\title{
Universal scaling of the critical temperature for thin films near the superconducting-to-insulating transition
}

\author{
Yachin Ivry, ${ }^{1, *}$ Chung-Soo Kim, ${ }^{1}$ Andrew E. Dane, ${ }^{1}$ Domenico De Fazio, ${ }^{1,2}$ Adam N. McCaughan, ${ }^{1}$ \\ Kristen A. Sunter, ${ }^{1}$ Qingyuan Zhao, ${ }^{1}$ and Karl K. Berggren ${ }^{1, \dagger}$ \\ ${ }^{1}$ Research Laboratory of Electronics, Massachusetts Institute of Technology, 77 Massachusetts Avenue, \\ Cambridge, Massachusetts 02139, USA \\ ${ }^{2}$ Department of Electronics and Telecommunications (DET), Polytechnic University of Turin, Corso Duca degli Abruzzi 24, 10129 Torino, Italy
}

(Received 22 July 2014; revised manuscript received 12 September 2014; published 15 December 2014)

\begin{abstract}
Thin superconducting films form a unique platform for geometrically confined, strongly interacting electrons. They allow an inherent competition between disorder and superconductivity, which in turn enables the intriguing superconducting-to-insulating transition and is believed to facilitate the comprehension of high- $T_{c}$ superconductivity. Furthermore, understanding thin film superconductivity is technologically essential, e.g., for photodetectors and quantum computers. Consequently, the absence of established universal relationships between critical temperature $\left(T_{c}\right)$, film thickness $(d)$, and sheet resistance $\left(R_{s}\right)$ hinders both our understanding of the onset of the superconductivity and the development of miniaturized superconducting devices. We report that in thin films, superconductivity scales as $d T_{c}\left(R_{s}\right)$. We demonstrated this scaling by analyzing the data published over the past 46 years for different materials (and facilitated this database for further analysis). Moreover, we experimentally confirmed the discovered scaling for $\mathrm{NbN}$ films, quantified it with a power law, explored its possible origin, and demonstrated its usefulness for nanometer-length-scale superconducting film-based devices.
\end{abstract}

DOI: 10.1103/PhysRevB.90.214515 PACS number(s): 74.78.-w, 74.70.-b, 64.70.Tg, 85.25.Oj

Relationships between low-temperature and normal-state properties are crucial for understanding superconductivity. For instance, the Bardeen-Cooper-Schrieffer theory (BCS) successfully associates the normal-to-superconducting transition temperature, $T_{c}$, with material parameters, such as the Debye temperature $\left(\Theta_{D}\right)$ and the density of states at the Fermi level $[N(0)]$. Hence, the BCS model allows us to infer superconducting characteristics (i.e., $T_{c}$ ) from properties measured at higher temperatures [1]. In the BCS framework, superconductivity occurs when attractive phonon-mediated electron-electron interactions overcome the Coulomb repulsion, giving rise to paired electrons (Cooper pairs) with a binding energy gap: $\Delta$. Moreover, within a superconductor, all Cooper pairs are coupled, giving rise to a collective electron interaction. Such a collective state is described by a complex global order parameter with real amplitude $(\Delta)$ and phase $(\varphi): \Psi=\Delta e^{i \varphi}$.

Because superconductivity relies on a collective electron behavior, the onset of superconductivity occurs when the number of participating electrons is just enough to be considered collective, i.e., at the nanoscale [2-5]. Thus, it is known that the superconductivity-disorder interplay varies in thin films and is effectively tuned with the film thickness $(d)$ or with the disorder in the system, which is represented by sheet resistance of the film at the normal state $\left(R_{S}\right)$ [6-10]. The mechanism of superconductivity in thin films has been investigated since the 1930s [6]. The development of thin film growth methods in the late 1960s allowed Cohen and Abeles [11] to demonstrate an

\footnotetext{
*Corresponding author: berggren@mit.edu

${ }^{\dagger}$ Corresponding author: ivry@ mit.edu
}

Published by the American Physical Society under the terms of the Creative Commons Attribution 3.0 License. Further distribution of this work must maintain attribution to the author(s) and the published article's title, journal citation, and DOI. increase in $T_{c}$ with decreasing thickness in aluminum films in a study that pioneered the currently ongoing research of thin superconducting films. This enhancement of $T_{c}$, which is still not completely understood, was later confirmed by Strongin et al. [12], who also reported the more common behavior of $T_{c}$-its suppression with reduced film thickness. Strongin et al. empirically examined different scaling options for the observed suppression of $T_{c}$ in lead and suggested that $T_{c}$ scales with $R_{s}$ better than it does with the other parameters, such as the film thickness. This suggestion is still influential on the data analysis done in the field today, and it encouraged the derivation of theoretical models to explain a dependence of $T_{c}$ on $R_{s}$. Indeed, Beasley et al. [8] (followed by Halperin and Nelson [13]) derived that $T_{c}$ depends only on $R_{s}$ for a Berezinsky-Kosterlitz-Thouless (BKT) transition, in which vortex-antivortex pairs, and not Cooper pairs, dominate the transition, which in turn is universal in nature. In addition, the mathematical derivation of the interacting-boson treatment within the BKT framework was also used in a reminiscent framework that associates interacting paddles of Cooper pairs with a multiple-Josephson junction array [14]. Likewise, Finkel'stein [9] used renormalization group tools to derive exactly a different expression for the dependence of $T_{c}$ on $R_{s}$ (with no direct dependence on the thickness). This derivation was based on a modified BCS equation, in which mean field theory was integrated with homogeneous disorder, i.e., impurity scattering due to Coulomb and spin density interactions. As opposed to these three models, which suggest that $T_{c}$ depends merely on $R_{s}$, competing models, such as the proximity effect [7] and the quantum size effect [10] theories, suggest that $T_{c}$ depends on $d$ only, with no direct dependence on $R_{s}$. Nevertheless, none of these models is sufficient to explain the entirety of the accumulated experimental data [11,12,15-28], despite the long-standing attempt to do so either through a direct mathematical derivation as in the above model, or with the aid of empirical universal laws [29]. 
Relationships between $d, R_{s}$, and $T_{c}$ are significant even to a broader scope than thin superconducting films. That is, the dependencies $T_{c}(d)$ and $T_{c}\left(R_{s}\right)$ are important for superconducting films. However, the dependence of resistivity $\left(\rho \equiv d R_{s}\right)$ on film thickness in thin metallic films has also occupied both scientists and technologists for many decades. Because above $T_{c}$, superconducting films behave like normal metals, the relationship $R_{s}(d)$ is similar to that of normal metals. That is, presumably, the resistivity is expected to remain constant or to demonstrate a smooth minor monotonic increase with reduced film thickness. Therefore, it is not important if $T_{c}$ is expressed as a function of $d$ or of $R_{S}$, as presumably, one parameter can be replaced by the other straightforwardly. Theoretically, the relationship $\rho(d)$ is usually discussed in terms of derivatives of Fuchs's theory [30], sometimes combined with Matthiessen's rule [31]. However, surprisingly, to date, the existing theories encounter difficulties in fitting the experimental data, which are often scattered when plotted on a $\rho(d)$ graph [32]. In addition to challenging our understanding of metallic thin films, such scatter prevents a smooth quantitatively valuable transition between descriptions of $T_{c}\left(R_{s}\right)$ and $T_{c}(d)$ in the case of superconductors.

An experimental work by Goldman et al. [20] suggested that beyond certain film thickness or sheet resistance values, $T_{c}$ is suppressed so much that, practically, the material will never become superconducting. That is, the cooling curves of such thin films indicate that $R_{S}$ increases with decreasing temperature - a behavior that is typical in insulators and not in metals. This observation began the race to understanding the superconductor insulator phase transition, which is believed by many researchers to be of a quantum nature [5]. To date, although much data for thin film superconductivity have been accumulated [11,12,15-28], and the local disorder has already been observed directly [33], the mechanisms governing the collective behavior close to the superconducting-to-insulating transition, or near the onset of superconductivity have remained elusive. That is, a model equivalent to the BCS, but that is valid for thin films, is still missing. Specifically, the theories that suggest that $T_{c}$ varies with either $R_{S}$ or $d$ are material dependent; whereas for some materials, none of the existing theories agrees with the observations. The absence of a unified description of superconductivity in thin films is even more pronounced when bearing in mind that the onset of superconductivity in such geometries is believed to occur through a quantum phase transition, which is in principle universal. Moreover, understanding superconductivity in thin films is expected to clarify the behavior of resistance in thin metallic films in general. Likewise, it has even been suggested that the superconductivity-disorder interplay in thin films is the key to understanding high- $T_{c}$ superconductivity [4]. Therefore, it is the goal of this paper to demonstrate a universal behavior for $T_{c}$ in thin films as a function of both $d$ and $R_{s}$.

In addition to the scientific impact associated with understanding superconductivity in thin films, thin superconducting films are of great technological significance because they are the basis for most miniaturized superconducting devices [34,35]. In particular, quantum-based technologies, such as computation, encryption, and communication rely on such films. Similarly, the leading technology for sensing single photons rapidly [36] and at a broad spectral range [37] - superconducting nanowire single photon detectors (SNSPDs) - is also based on thin superconducting films [34]. Nevertheless, the lack of understanding of the underlying mechanism of superconductivity in thin films and the large scatter of the experimental data for the relationships between $T_{c}, R_{s}$, and $d$ typically lead to low confidence in the film growth process, encumbering the relevant technological developments. Specifically, the limited reproducibility and control of the film parameters impair both the yield and the size of devices made out of such films. For instance, the yield of SNSPDs made out of thin niobium nitride $(\mathrm{NbN})$ films is low, while their active area is usually restricted, hindering the technological advances in the field. Hence, a universal scaling of the properties of thin superconducting films is expected to improve the control and reproducibility of the film properties and, therefore, to allow at last realization of the potential of miniaturized superconducting devices.

We show that for a given material, the relationship between film thickness, sheet resistance, and critical temperature scales as $d T_{c}\left(R_{s}\right)$. Moreover, this scaling typically follows a power law. We demonstrated the scaling on data gathered from 30 different sets of materials published since 1968, which cover most of the literature. The materials studied included clean, dirty, granular, and amorphous superconductors. Some of these materials are type I in their bulk state, and some are type II. Most of these materials exhibited suppression of $T_{c}$ at reduced thicknesses, but some exhibited enhancement. The data in its entirety could not fit previous theories [7-10], but the data set did fit the new power-law relationship across a broad range of $T_{c}, d$, and $R_{s}$. We extracted the coefficient and exponent of the power law for each material, and we demonstrated that the coefficient and the exponent are correlated. The power law fits the data from materials that also fit one of the previous models $T_{c}(d)$ or $T_{c}\left(R_{s}\right)$, as well as for materials that presumably are not BCS. We also examined our own new experimental data on $\mathrm{NbN}$ thin films. In these data, relating $d T_{c}$ to $R_{s}$ provided fits with reduced scatter relative to fits suggested by previous models [7-10,30-32]. Finally, we supply two possible explanations of the observed universal behavior. We should note that the data gathered from the literature are available for further review in the Supplemental Material [38].

To illustrate the new scaling, we will start by examining our data on $\mathrm{NbN}$ films. We chose sputtered $\mathrm{NbN}$ as the material for this study for four main reasons: (a) It is widely researched, and experimental data collected for different growth methods and conditions are available; (b) there are contradicting reports about which of the existing models describes the $T_{c}$ suppression in $\mathrm{NbN}$ films-for instance, Kang et al. [18] proposed that $T_{c}$ is suppressed due to the quantum size effect, Wang et al. [17] suggested that the suppression follows Finkel'stein's model [9], Semenov et al. [19] determined that the suppression is governed by the proximity effect, and Koushik et al. [39] argued that the transition is of a BKT type; (c) the relatively high $T_{c}$ of $\mathrm{NbN}$ (16 K for a bulk NbN [40]) assists the experimental investigation; and (d) its properties make it useful for photodetectors [34,36,41,42].

Figures 1(a) and 1(b) show the dependence of $T_{c}$ on thickness and on sheet resistance for our $\mathrm{NbN}$ films, allowing a comparison of the data with the existing models [7-10,30-32]. Although a general trend can be seen in both $T_{c}(d)$ and $T_{c}\left(R_{s}\right)$, 

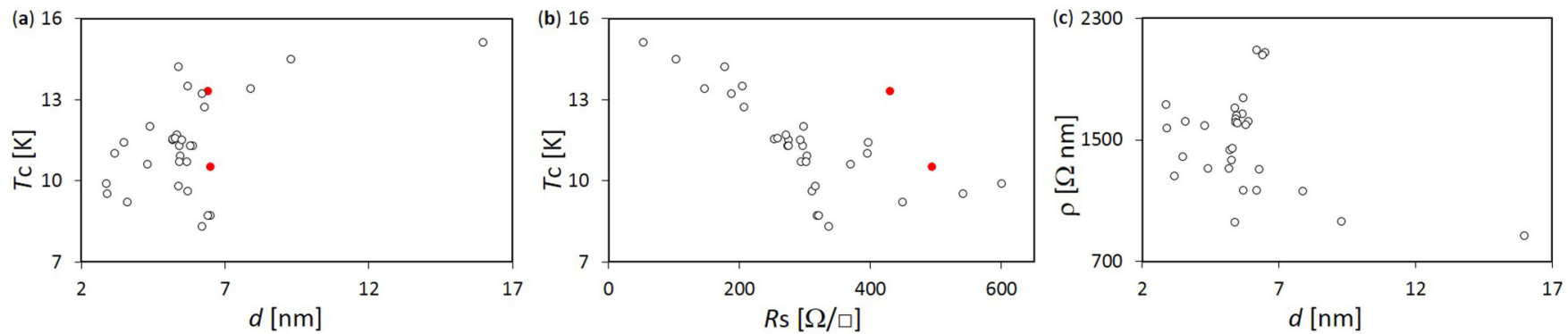

FIG. 1. (Color online) Metallic and superconducting behavior of thin $\mathrm{NbN}$ films. (a) Critical temperature of NbN films as a function of the film thickness $(d)$ and (b) sheet resistance $\left(R_{s}\right)$ indicate no clear correlation with general functions of the form $T_{c}\left(R_{s}\right)$ or $T_{c}(d)$. (c) Resistivity $\left(\rho \equiv R_{s} d\right)$ of the $\mathrm{NbN}$ films vs thickness reveals a scattered data set. Chemical treatment of the substrate prior to deposition is suspected of influencing the properties of the red solid points here and in Fig. 3, while their high resistivity is outside the range presented in (c) but is discussed in the Supplemental Material [38].

the scatter in these graphs is too large to allow confident fitting to any model of the form $T_{c}(d)$ or $T_{c}\left(R_{s}\right)$. Bearing in mind the metallic characteristic of the films, the resistivity of the grown films corresponds to their inverse mean free path and hence should increase monotonically with decreasing thickness [30]. However, Fig. 1(c) shows the dependence of resistivity on thickness in our films, revealing again, large scatter of the data points with only vaguely the expected trend. (As a side remark, we should note that the regime in which the scatter is the largest is when the thickness is around $6 \mathrm{~nm}$, which in turn is the nominal coherence length of NbN films [19].)

One potential possible origin for the large scatter and for the deviation from a clear trend of the curves $T_{c}(d)$ or $T_{c}\left(R_{s}\right)$ in our $\mathrm{NbN}$ films is low material quality, which might stem, e.g., from extensive granularity, poor crystallinity, large strain, etc. To avoid such effects and to obtain high material quality, we grew the $\mathrm{NbN}$ films on $\mathrm{MgO}$ substrates, with which the lattice mismatch is small $(<3.5 \%)$. Moreover, during the deposition, we heated the substrate to a nominal temperature of $800{ }^{\circ} \mathrm{C}$ to further improve the crystallographic growth by relaxing the deposited film. We also used a system with a low base pressure (4.5-9 $\times 10^{-9}$ Torr), minimizing the magnetic and other impurities in the films. To determine the quality of our $\mathrm{NbN}$ films, we used transmission electron microscopy (TEM) and demonstrated [Fig. 2(a)] that our $\mathrm{NbN}$ films are grown epitaxially on the $\mathrm{MgO}$ substrate, forming highly orientated crystallinity, i.e., clear long-range cubic structure with low levels of granularity, if any exist at all (we examined representative films with TEM; see Appendix for details). In addition to the atomic resolution TEM imaging, the highly orientated crystallinity of the $\mathrm{NbN}$ films and the good lattice matching between the $\mathrm{NbN}$ and the $\mathrm{MgO}$ substrate were observed also in the selective area electron diffraction [Fig. 2(b)]. Furthermore, the measured lattice constant of the cubic $\mathrm{NbN}$ films is very close to the literature value, suggesting the films are relaxed.

Given that the epitaxial films were of high quality and that they were grown under similar conditions, the fact that Fig. 1 failed to show any clear correlations between the parameters $d$, $R_{s}$, and $T_{c}$ suggests that a different scaling method is required. Because $T_{c}$ is usually suppressed with reduced thickness and with increase in disorder (i.e., with increasing $R_{s}$ ), we examined the relationship $d T_{c}$ as a function of $R_{s}$. Indeed, Fig. 3 shows that plotting $d T_{c}$ vs $R_{s}$ reveals a clear trend, while the scatter was reduced significantly with respect to the traditional scaling curves that were presented in Fig. 1. This decrease in scatter is even more remarkable when taking into account that when multiplying two parameters that were measured independently (i.e., thickness and $T_{c}$ ), the statistical noise should increase and not decrease.

The blue solid line in Fig. 3 was added not only to guide the eye for the clear trend and reduced scatter with respect to Fig. 1, but this line is also the best fit of the data to the power law:

$$
d T_{c}=A R_{s}^{-B}
$$

where $A$ and $B$ are fitting parameters and hereafter $d, T_{c}$, and $R_{s}$ are unitless when the appropriate values are given in nanometers, Kelvin, and ohms per square. The exponent $B$ in Fig. 3 is close to unity ( $B \approx 0.9$ ), so technically, one can approximate Eq. (1) to a reduced form: $\rho T_{c} \sim$ constant. Yet, when using Eq. (1) to predict the $T_{c}$ of a film [38], the exponent $B$ is needed.

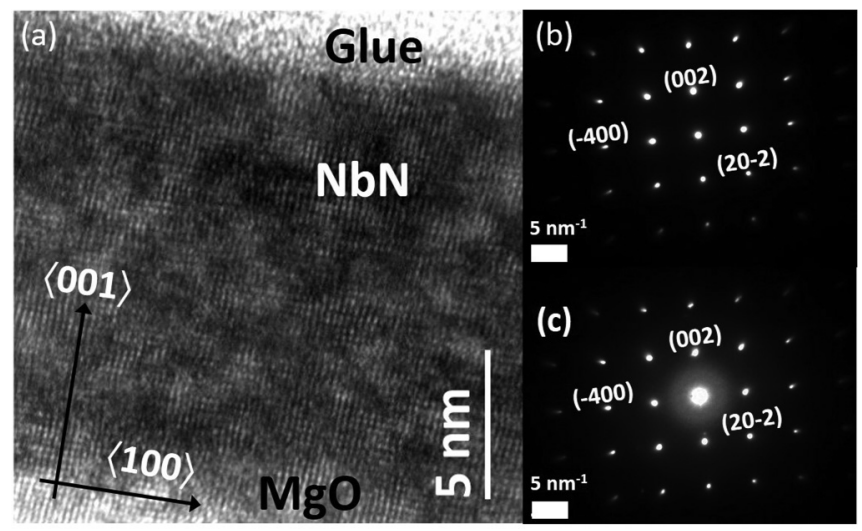

FIG. 2. TEM micrographs of epitaxial $\mathrm{NbN}$ film on an $\mathrm{MgO}$ substrate. (a) Atomic structure of an $\mathrm{NbN}$ film on an $\mathrm{MgO}$ substrate, demonstrating epitaxial growth of long-range cubic structure and good lattice matching with the substrate. The lattice constants of both $\mathrm{MgO}$ and $\mathrm{NbN}$ are $4.35 \pm 0.1 \AA$. (b) Selective area electron diffraction from an area within the $\mathrm{MgO}$ substrate only and (c) from an area that spans the $\mathrm{MgO}$ substrate, $\mathrm{NbN}$ film, and the glue layer (that is used to protect the film from the top) demonstrates high crystallinity of both the $\mathrm{MgO}$ and $\mathrm{NbN}$ and a good lattice matching between these substances. The bright spot in the center of (c) is due to the amorphous glue. 


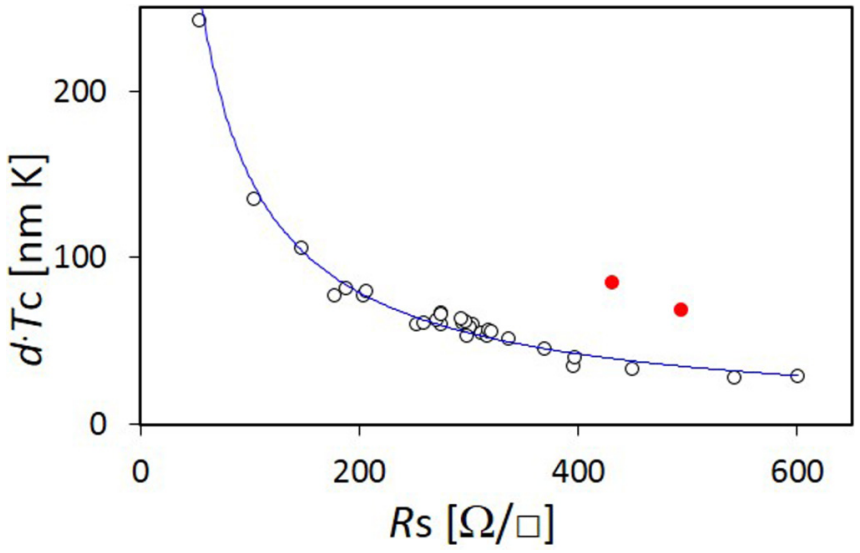

FIG. 3. (Color online) Fitting our $\mathrm{NbN}$ on $\mathrm{MgO}$ data to the power law $d T_{c}\left(R_{s}\right)$. Plotting $d T_{c}$ vs $R_{s}$ for the $\mathrm{NbN}$ films reduces the scattering significantly with respect to the curves in Fig. 1 . The blue line is the best fit to Eq. (1) $(A=9448.1$ and $B=0.903)$. Red solid points are discussed in the Supplemental Material [38].

We can suggest two approaches to explain the origin of Eq. (1). The first approach is based on the BCS-related models. Specifically, one can rewrite Eq. (1) as:

$$
T_{c}=(A / d) e^{-B \ln \left(R_{\mathrm{s}}\right)}
$$

Bearing in mind the BCS-based frameworks, Eq. (2a) is written in a similar form to these equations, i.e., $T_{c}$ is equal to an amplitude $(A / d)$ times an exponent that expresses the electron interactions $\left[B \ln \left(R_{S}\right)\right]$. For instance, in the framework of the BCSbased McMillan equation $\left(T_{c}=\frac{\Theta_{D}}{1.45} e^{-\frac{1.04(1+\lambda)}{\lambda-\mu^{*}(1+0.62 \lambda)}}\right)[43,44]$, Eq. (2a) implies that changes in $N(0)$ or in the interaction ( $\lambda$ or $\mu$ ) may scale as $B \ln \left(R_{s}\right)$ (where $\lambda$ and $\mu$ are the electron-phonon coupling constant and the Coulomb repulsive interactions, respectively). This outcome is reminiscent also of Finkel'stein's [9] derivation of $T_{c}\left(R_{S}\right)$ for homogeneous superconductors, where the interaction term was also rephrased in terms of the sheet resistance (while we recall that a logarithmic accuracy was claimed in that framework), but with the main difference being that here $d$ appears explicitly.

The second approach to explain Eq. (1) relies on the fact that above $T_{c}$, conventional superconductors are normal metals. Thus, the relationship between $d$ and $R_{s}$ for the examined thin superconducting films is the same as that for metals in general. Hence, here, Eq. (1) implies a somewhat broader generalization of the thickness dependence of resistance in thin metallic films. That is, one can isolate $R_{s}$ as a function of $d$ and $T_{c}$ :

$$
R_{\mathrm{s}}=\left(A / d T_{c}\right)^{1 / B}
$$

This manipulation is justified, e.g., if $A, B$, and $T_{c}$ are representatives of simple material properties such as $\Theta_{D}$, mechanical strain, granularity, $N(0)$, etc. In this case, a power-law-form thickness dependence of these properties can also explain Eq. (1). We should emphasize here that although for thick materials, $R_{s} \sim 1 / d$, this is usually not true for thin films (i.e., when $T_{c}$ deviates significantly from its bulk value), as can be seen for instance in Fig. 1(c). Therefore, the fit of our data to Eq. (1) (Fig. 3) cannot be due to such simple relationships.
It is worth mentioning that Eq. (1), and more so its reduced form, resembles Homes's law, which empirically relates $T_{c}$ through the superfluid density to the normal state conductivity in the case of high- $T_{c}$ superconductors [3]. However, thus far, we have not been able to derive a direct relationship between the two laws.

To demonstrate the full range of applicability of Eq. (1), we show that this equation fits data gathered from the literature for $\sim 30$ other superconductors studied over the past 46 years that summarize all of the reports from which we could extract $d, R_{s}$, and $T_{c}$ [11,12,15-28]. In some cases, we merged data reported in different publications by the same authors. We should note that, although $R_{s}$ can usually be measured rather accurately, the thickness, which is measured indirectly, is typically reported with a lower level of confidence [38]. Moreover, although there is an ongoing dispute of how to determine $T_{c}$ in thin films, usually the values for $T_{c}$ are measured in a consistent manner within a data set of a given material, allowing an examination of each data set at least with itself [45]. These data include $\mathrm{NbN}$ sets of films that were reported previously by other groups, each of which was reported to be in agreement with one of the models of the form $T_{c}\left(R_{s}\right)$ [17] or $T_{c}(d)$ [18,19]. Moreover, these data sets include some "classical" examples, such as the Bi films by Goldman et al. [20], which were used for demonstrating a possible superconducting-to-insulating transition, and the homogeneous $\alpha$ MoGe films of Graybeal et al. [46], which were used for demonstrating Finkel'stein's model [9]. The data and analysis of each of these materials are discussed in detail on a linear scale in the Supplemental Material [38] (e.g., a detailed analysis of $\alpha \mathrm{MoGe}$ films is provided in section S7 in the Supplemental Material [38]). We should note that in addition to the contribution of the Supplemental Material [38] to the current paper, this inclusive database is available also for readers who seek further investigation of superconducting and metallic behavior in thin films.

Although a detailed analysis of the individual materials is provided in the Supplemental Material [38], the most common method to present data points that follow Eq. (1) is by linearity on a log-log scale of $d T_{c}$ vs $R_{s}$. Indeed, the linearity of the data in Figs. 4(a) and 4(b) clearly validates Eq. (1) for a broad range of $T_{c}, d$, and $R_{s}$. [We divided the data between Figs. 4(a) and 4(b) arbitrarily to spread data that otherwise would have been too crowded to distinguish.] To eliminate the possibility that the scaling of Eq. (1) is due to, e.g., an inverse proportionality between $R_{s}$ and $d$, which by chance fits with a power-law relation for $T_{c}\left(R_{s}\right)$ or for $T_{c}(d)$, in Fig. 4(c), we present the resistivity as a function of thickness for these materials. Likewise, in Figs. 4(d) and 4(e), we show the dependence of $T_{c}$ on thickness and on sheet resistance. The nonlinearity and nonuniformity of the data in Figs. 4(c)-4(e) emphasize the universality presented in Figs. 4(a) and 4(b). (We should note that the set of $\alpha \mathrm{Nb}_{3} \mathrm{Ge}$ films reported by Kes and Tsuei [23] and the thicker films of Wang et al. [15-17] are given as examples for thick films, in which both the resistivity and $T_{c}$ are rather constants over the large range of thicknesses reported for these films. However, it is clear from Fig. 4 that this is not the case for all the other data sets.) In addition, the complete presentation of the individual sets of data on a linear scale in the Supplemental Material [38] 


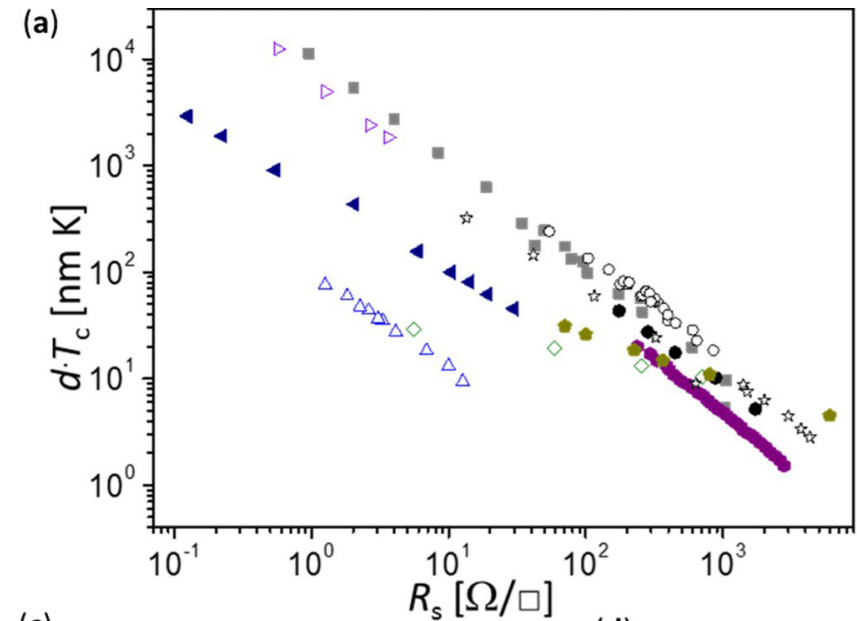

(c)

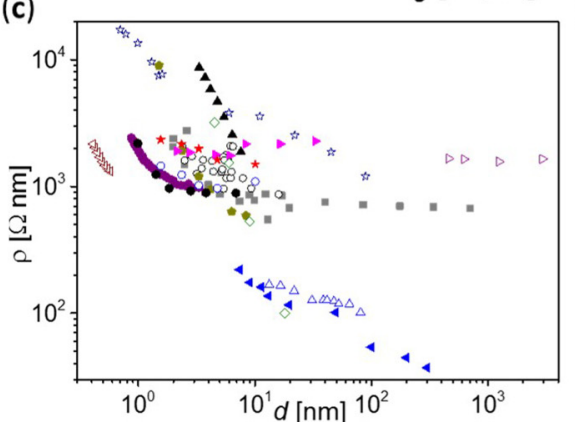

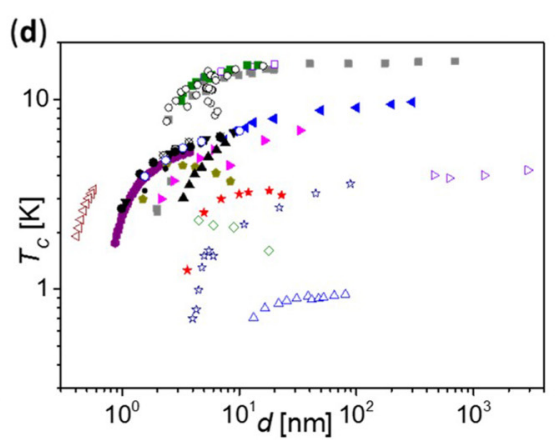

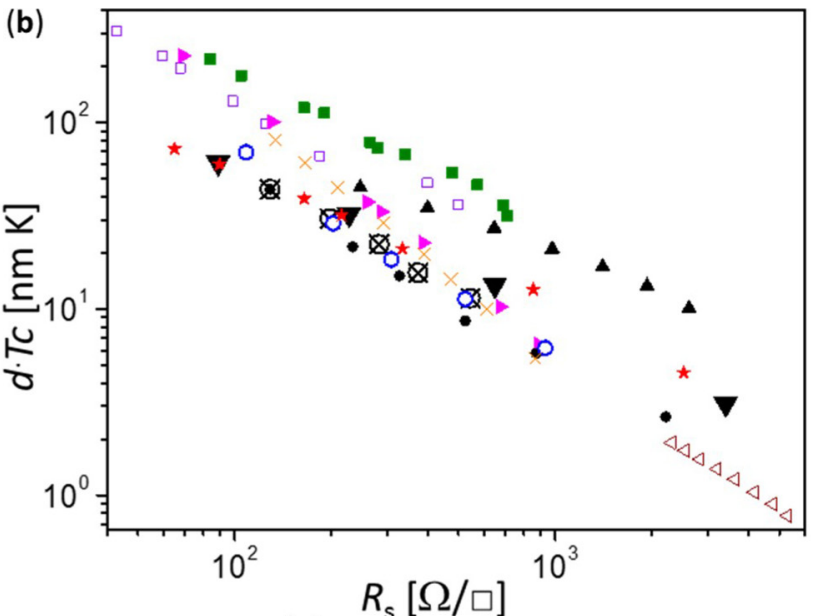

(e)

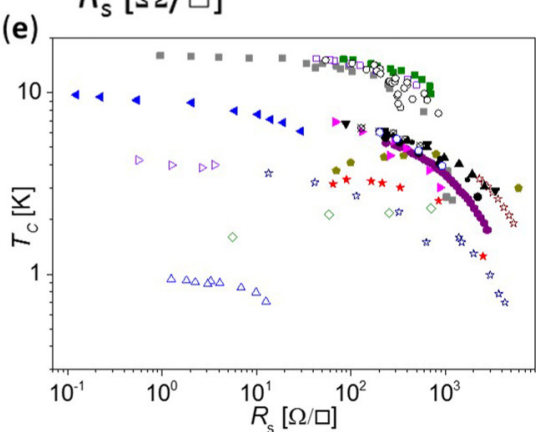

FIG. 4. (Color online) Scaling of superconducting and metallic properties in thin films. (a) and (b) are the dependences of $d T_{c}$ on $R_{s}$ for various superconductors (data sets are arbitrarily split into two panels to prevent indistinguishability). The linearity of the data on a log-log scale is in good agreement with Eq. (1). (c) Resistivity dependence on thickness for representative materials (log-log scale). (d) $T_{c}$ as a function of thickness and (e) of sheet resistance of these materials on log-log scales. The following symbols were used in (a-e): $-\mathrm{NbN}$ from Wang et al. [15-17]; - -NbN by Semenov et al. [19]; $\square-N b N$ by Kang et al. [18]; $\bigcirc$-our NbN films (from Fig. 1); $\triangle$-Mo [50]; $\square$-Bi and $\backslash$-Pb by

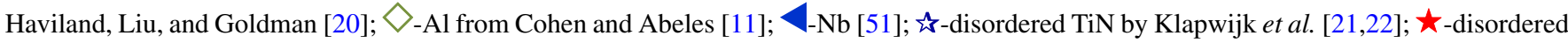
TiN by Baturina et al. [52]; $\searrow_{-} \alpha \mathrm{Nb}_{3}$ Ge [23]; X- $\alpha$ MoGe from Graybeal and Beasley [53]; $-\alpha$ MoGe from Graybeal et al. [24-26]; $-\alpha$ MoGe

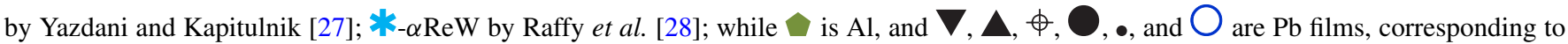
the same symbols used by Strongin et al. [12]. A complete list of the data is given in the Supplemental Material [38].

demonstrates that Eq. (1) quantitatively fits well with the data from each material. This list, which surveys thin-film superconductors and presents their properties, also includes some superconductors that require more gentle treatment, for instance, superconductors that only qualitatively agree with the scaling $d T_{c}$ vs $R_{s}$ (e.g., $\mathrm{MgB}_{2}$ [47]), as well as the few material sets that do not exhibit convincing agreement with this scaling ( $\mathrm{Ga}$ [48], $\mathrm{Sn}$ [12], $\mathrm{Nb}_{3} \mathrm{Sn}$, and $\mathrm{V}_{3} \mathrm{Si}$ [49]).

To allow further examination of the universality presented in Figs. 4(a) and 4(b), we plotted in Fig. 5(a) the intercepts of the different curves as a function of their slopes [ $A$ vs $B$ in Eq. (1)]. In this way, each material is represented by a single data point, allowing a comparison between the different superconductors. Figure 5(a) shows that the data points follow a general trend, so that $A$ and $B$ are correlated. It is interesting to note that the materials at the two extreme points of this curve are aluminum (in which $T_{c}$ is enhanced in thin films) and $\alpha$ MoGe, implying that $A$ and $B$ may be determined by the granularity of the superconductor. In fact, since the interaction in Eq. (2a) is reminiscent of Finkel'stein's model [9], which in turn had no implicit dependence on thickness and is valid for homogeneous (amorphous) superconductors, Fig. 5(a) suggests that the thickness-dependent coefficient is more significant to granular films, while for amorphous films, the $R_{s}$-based interaction is dominating. Further discussion about the potential relationship between $A$ and $B$ can be found in the Supplemental Material (mainly in sections S7.1 and S17) [38]. Independently, the data aggregation around $B=1$ indicates that $\rho T_{c} \sim$ constant is a reasonable approximation in several cases. More specifically, the mean value of the histogram in Fig. 5(b) suggests that $B \approx 0.9$ to 1.1 is a universal exponent that represents the scaling of Eq. (1). In fact, a correlation between the coefficient and the exponent such as the one observed in Fig. 5(a) indicates that a logarithmic correction to the power law may support universality of the exponent $B \approx 0.95$. We should note that a universal value of $B$ (e.g., $B=$ 0.95 ) means that this may help in describing superconductivity in thin films in general, but it does not mean that such a value is a good approximation for any of the specific materials.

It often occurs that one or more films in a data set are different than the others, e.g., due to faults in the growth process. In many cases, it is difficult to identify such a film in a $T_{c}\left(R_{s}\right)$ or in a $T_{c}(d)$ curve. Therefore, the confidence in determining whether the growing system is stable or not is 

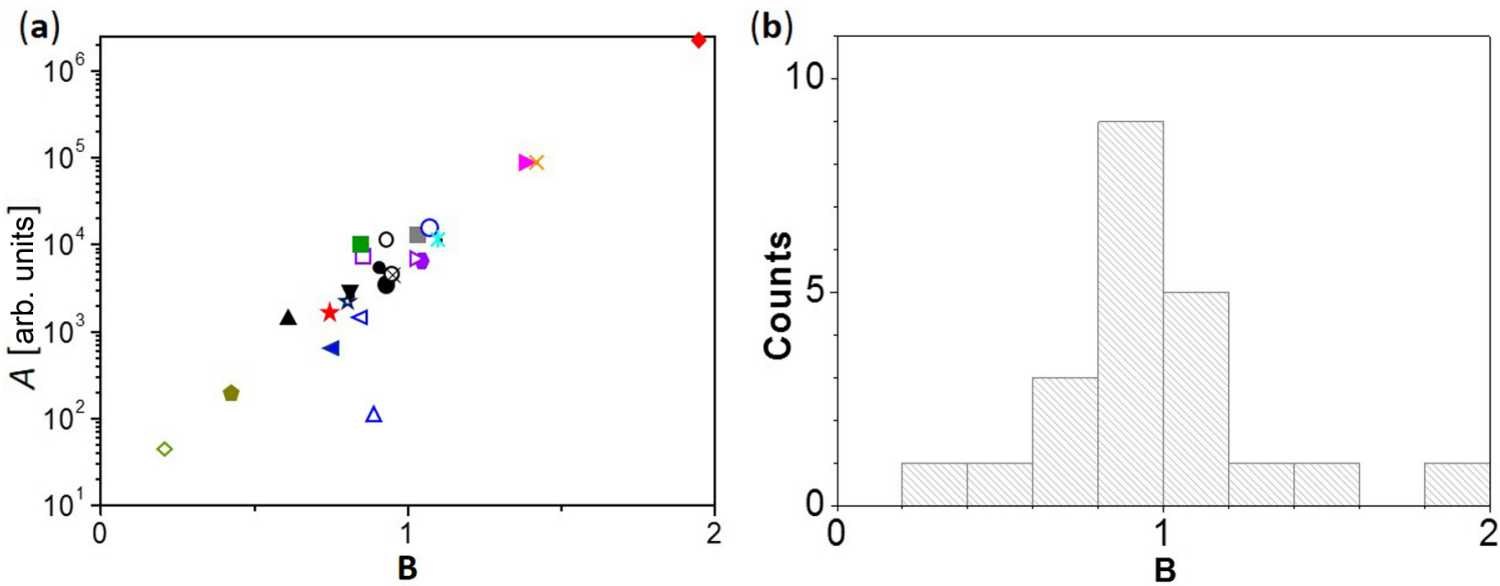

FIG. 5. (Color online) Universality of the scaling of $d T_{c}\left(R_{s}\right)$. (a) Plot of intercept vs slope [ $A$ vs $B$ with respect to Eq. (1)] of the best linear fits for the graphs in Figs. 4(a) and 4(b) suggests that the parameters $A$ and $B$ are correlated (for details, see S7 and S17 in the Supplemental Material [38]). Legend corresponds to Fig. 4, while the only material outside the trend $(\triangle)$ is molybdenum. (b) Histogram of the exponent $B$ of the different materials with a mean value at $B \approx 0.95$.

low. This lack of confidence obstructs the relevant scientific studies. Furthermore, it affects very badly the ability to reliably fabricate miniaturized superconducting devices. The solid red points in our own data in Figures 1 and 3 and two films from Semenov et al. [19] are examples, and they are discussed in detail in the Supplemental Material [38] (sections S11.5 and S11.2). Since such films stand out on a $d T_{c}\left(R_{s}\right)$ curve, we propose to use this scaling as a practical method to assess the quality of superconducting films. Moreover, once the parameters $A$ and $B$ in Eq. (1) are determined for a specific set of films, $T_{c}$ can be derived from measurements that are done in the normal state (e.g., at room temperature). Indeed, using the scaling law of Eq. (1), we were able to better control and evaluate our growth system. This control helped us in increasing the yield of SNSPDs made in our group. Moreover, the improvement of control of the film properties allowed us to make larger areas, and hence more advanced nano-superconducting devices [54,55]. In addition, the analysis done with Eq. (1) also proved useful to predict the behavior of thin TiN films, as discussed in section S15.1 in the Supplemental Material [38].

In conclusion, we showed that in thin superconducting films, close to the superconducting-to-insulating transition, the scaling $d T_{c}\left(R_{s}\right)$ describes the relationships between the film properties. We demonstrated this scaling for the films grown by us and showed that it fits our data better than the previously proposed scaling for $T_{c}$ in thin films. Moreover, by examining the data existing in the literature, we demonstrated the universality of this scaling. We quantified the scaling with a power law and supplied possible explanations of its origin. Furthermore, using this scaling, we presented a method to evaluate the quality of a grown film as well as to estimate its $T_{c}$, assisting in the control of thin superconducting films, and hence expediting the development of miniaturized superconducting devices and the research of superconductivity at low dimensions. In addition, because existing theories of metallic thin films relating $R_{s}$ (or $\rho$ ) and $d$ do not involve $T_{c}$, while our finding does, our result may facilitate better understanding of metallic thin films more generally. Finally, the inclusive database formed to allow quantitative analysis of the existing data from the literature [38] can be used for further investigation of universal behavior in thin superconductivity films.

The authors would like to thank Leonid S. Levitov, Bertrand I. Halperin, Teunis M. Klapijk, Patrick A. Lee, Yosef Imry, Terry P. Orlando, Eduard F. C. Driessen, Mehran Kardar, Karen Michaeli, Biabing Jin, Pieter Jan C. J. Coumou, Emanuele G. Dalla Torre, Baruch Barzel, and Richard G. Hobbs for very useful discussions. Moreover, they thank the Center for Excitonics, an Energy Frontier Research Center funded by the US Department of Energy, Office of Science, Basic Energy Sciences under Award No. DE-SC0001088 for funding the project and Y.I.; A.E.D., and K.A.S. as well as the test apparatus and growth were supported by IARPA (Award No. FA8650-11-C-7105), while A.M.C. acknowledges fellowship support from the National Science Foundation iQuISE program, Award No. 0801525. The TEM experiment was supported by the U.S. Office of Naval Research (Contract No. N00014-13-1-0074).

\section{APPENDIX: MATERIALS AND METHODS}

$\mathrm{NbN}$ films were sputtered with an ATC ORION sputtering system, EMOC-380 power distribution, and SHQ-15A PID heater controller from AJA International, Inc. Sputtering conditions were nominal temperature of $800^{\circ} \mathrm{C}$; a total pressure of 1.5-6 mTorr; $\mathrm{Ar}$ and $\mathrm{N}_{2}$ flows of $26.5 \mathrm{sccm}$ and 3-7.5 sccm, respectively; a sputtering current of $400 \mathrm{~mA}$; and a target-sample distance of $47 \mathrm{~mm}$. The sputtering time ranged from $45 \mathrm{~s}$ to $300 \mathrm{~s}$. We used 2 "diameter $\times 0.25$ " thick $\mathrm{Nb}$ 99.95\% ExTa targets from Kurt J. Leskor and $10 \times 10 \times$ $0.5 \mathrm{~mm}^{3}\langle 100\rangle \mathrm{MgO}$ substrates with both sides polished. $R_{s}$ was extracted at ambient conditions from standard four-probe measurements with a Remington Test LCC stage and a Keithley 2400 SourceMeter. $T_{c}$ was determined as the temperature at which $R_{s}=\left[0.9 R_{S}(@ 20 K)+0.1 R_{s}(@ 20 K)\right] / 2$, where $R_{s}(@ 20 \mathrm{~K})$ is the measured sheet resistance at $20 \mathrm{~K}$. 
$T_{c}$ was measured in liquid $\mathrm{He}$ with an in-house made dipstick (Omegalux KHLV-102/10 flexible heater, a DT-670A LakeShore temperature sensor, and a Cryo-con34 temperature controller). Finally, $d$ values were measured with an inhouse made reflectometer (photodiodes: ThorLabs DET 36A biased detectors, 350-1100 nm wavelength; LED: $470 \mathrm{~nm}$ HI VIS TO -5 IDX:1 OptoDiode Corp. 00-469L-ND; LED Driver: ThorLabs LEDD1B; and Hewlett Packard 34401A Multimeter). The TEM images were taken with JEM 2010F by JEOL with a $200 \mathrm{kV}$ beam for several samples from different locations from two films of $14.2 \mathrm{~nm}$ and $2.5 \mathrm{~nm}$; all were found to share a similar structure. The selective area electron diffraction images were in great agreement with the fast Fourier transform of the atomic images taken from the same areas. Data presented here are from a film with $d=$ $14.2 \mathrm{~nm}$ as measured with the reflectometer $(14.6 \mathrm{~nm}$ extracted from the TEM image), $R_{s}=75.69 \Omega / \square$, and $T_{c}=14.24 \mathrm{~K}$.
The film was sputtered while being held with a thinner sample holder than that used for the set of films presented in Figs. 1 and 3 , potentially giving rise to a slightly higher substrate temperature.

Previously published data were collected with DataThief III version 1.6 [56]. Whenever the data were collected from several different sources (i.e., from different figures within the same work), cross-checking was done, and data points were discarded when the inconsistency was large. Moreover, data were also discarded when it was stated clearly by the authors that the films were too thin to be continuous or to allow reliable measurements (some of the published data were sent by the authors of these publications). A complete list of the collected and presented data, and the cross-checking and discussion of each data set are given in the Supplemental Material [38] (which also includes the works presented in references [57-67]).
[1] M. Tinkham, Introduction to Superconductivity, 2nd ed. (Dover Publications, New York, 2004), p.454.

[2] S. Chakravarty, A. Sudbø, P. W. Anderson, and S. Strong, Science 261, 337 (1993).

[3] C. C. Homes, S. V. Dordevic, M. Strongin, D. A. Bonn, R. Liang, W. N. Hardy, S. Komiya, Y. Ando, G. Yu, N. Kaneko, X. Zhao, M. Greven, D. N. Basov, and T. Timusk, Nature 430, 539 (2004).

[4] Y. Dubi, Y. Meir, and Y. Avishai, Nature 449, 876 (2007).

[5] V. F. Gantmakher and V. T. Dolgopolov, UFN 180, 3 (2010) [Phys.-Usp. 53, 1 (2010)].

[6] A. Shalnikov, Nature 142, 74 (1938).

[7] L. N. Cooper, Phys. Rev. Lett. 6, 689 (1961).

[8] M. Beasley, J. Mooij, and T. Orlando, Phys. Rev. Lett. 42, 1165 (1979).

[9] A. M. Finkel'stein, Phys. B Condens. Matt. 197, 636 (1994).

[10] Y. Guo, Y.-F. Zhang, X.-Y. Bao, T.-Z. Han, Z. Tang, L.-X. Zhang, W.-G. Zhu, E. G. Wang, Q. Niu, Z. Q. Qiu, J.-F. Jia, Z.-X. Zhao, and Q.-K. Xue, Science 306, 1915 (2004).

[11] R. W. Cohen and B. Abeles, Phys. Rev. 168, 444 (1968).

[12] M. Strongin, R. Thompson, O. Kammerer, and J. Crow, Phys. Rev. B 1, 1078 (1970).

[13] B. Halperin and D. Nelson, Phys. Rev. Lett. 41, 121 (1978).

[14] M. W. Johnson and A. M. Kadin, Phys. Rev. B 57, 3593 (1998).

[15] Z. Wang, A. Kawakami, Y. Uzawa, and B. Komiyama, J. Appl. Phys. 79, 7837 (1996).

[16] S. Miki, Y. Uzawa, A. Kawakami, and Z. Wang, Electron. Commun. Japan Part II Electron. 85, 77 (2002).

[17] S. Ezaki, K. Makise, B. Shinozaki, T. Odo, T. Asano, H. Terai, T. Yamashita, S. Miki, and Z. Wang, J. Phys. Condens. Matt. 24, 475702 (2012).

[18] L. Kang, B. B. Jin, X. Y. Liu, X. Q. Jia, J. Chen, Z. M. Ji, W. W. Xu, P. H. Wu, S. B. Mi, A. Pimenov, Y. J. Wu, and B. G. Wang, J. Appl. Phys. 109, 033908 (2011).

[19] A. Semenov, B. Günther, U. Böttger, H.-W. Hübers, H. Bartolf, A. Engel, A. Schilling, K. Ilin, M. Siegel, R. Schneider, D. Gerthsen, and N. Gippius, Phys. Rev. B 80, 054510 (2009).

[20] D. B. Haviland, Y. Liu, and A. M. Goldman, Phys. Rev. Lett. 62, 2180 (1989).

[21] E. F. C. Driessen, P. C. J. J. Coumou, R. R. Tromp, P. J. de Visser, and T. M. Klapwijk, Phys. Rev. Lett. 109, 107003 (2012).
[22] P. C. J. J. Coumou, E. F. C. Driessen, J. Bueno, C. Chapelier, and T. M. Klapwijk, Phys. Rev. B 88, 180505(R) (2013).

[23] H. Kes and C. C. Tsuei, Phys. Rev. B 28, 5126 (1983).

[24] H. Tashiro, J. Graybeal, D. Tanner, E. Nicol, J. Carbotte, and G. Carr, Phys. Rev. B 78, 014509 (2008).

[25] S. Turneaure, T. Lemberger, and J. Graybeal, Phys. Rev. B 63, 174505 (2001).

[26] S. Turneaure, T. Lemberger, and J. Graybeal, Phys. Rev. B 64, 179901 (2001).

[27] A. Yazdani and A. Kapitulnik, Phys. Rev. Lett. 74, 3037 (2000).

[28] H. Raffy, R. Laibowitz, P. Chaudhari, and S. Maekawa, Phys. Rev. B 28, 6607 (1983).

[29] S. Wolf, D. Gubser, and Y. Imry, Phys. Rev. Lett. 42, 324 (1979).

[30] K. Fuchs and H. H. Wills, Math. Proc. Cambridge Philos. Soc. 34, 100 (1938).

[31] P. A. Badoz, A. Briggs, E. Rosencher, F. A. D'Avitaya, and C. D'Anterroches, Appl. Phys. Lett. 51, 169 (1987).

[32] T. Sun, B. Yao, A. P. Warren, K. Barmak, M. F. Toney, R. E. Peale, and K. R. Coffey, Phys. Rev. B 81, 155454 (2010).

[33] B. Sacépé, C. Chapelier, T. Baturina, V. Vinokur, M. Baklanov, and M. Sanquer, Phys. Rev. Lett. 101, 157006 (2008).

[34] G. N. Gol'tsman, O. Okunev, G. Chulkova, A. Lipatov, A. Semenov, K. Smirnov, B. Voronov, A. Dzardanov, C. Williams, and R. Sobolewski, Appl. Phys. Lett. 79, 705 (2001).

[35] J. Clarke and F. K. Wilhelm, Nature 453, 1031 (2008).

[36] F. Najafi, F. Marsili, E. Dauler, R. J. Molnar, and K. K. Berggren, Appl. Phys. Lett. 100, 152602 (2012).

[37] F. Marsili, F. Bellei, F. Najafi, A. E. Dane, E. A. Dauler, R. J. Molnar, and K. K. Berggren, Nano Lett. 12, 4799 (2012).

[38] See Supplemental Material at http://link.aps.org/supplemental/ 10.1103/PhysRevB.90.214515 for detailed discussion about the individual data sets, including for the raw data that were used to perform the analysis that is presented in the paper.

[39] R. Koushik, S. Kumar, K. R. Amin, M. Mondal, J. Jesudasan, A. Bid, P. Raychaudhuri, and A. Ghosh, Phys. Rev. Lett. 111, 197001 (2013). 
[40] B. Matthias, T. Geballe, and V. Compton, Rev. Mod. Phys. 35, 1 (1963).

[41] K. M. Rosfjord, J. K. W. Yang, E. A. Dauler, A. J. Kerman, V. Anant, B. M. Voronov, G. N. Gol'tsman, and K. K. Berggren, Opt. Express 14, 527 (2006).

[42] F. Marsili, F. Najafi, E. Dauler, F. Bellei, X. Hu, M. Csete, R. J. Molnar, and K. K. Berggren, Nano Lett. 11, 2048 (2011).

[43] W. McMillan, Phys. Rev. 167, 331 (1968).

[44] P. B. Allen, Phys. Rev. B 12, 905 (1975).

[45] T. I. Baturina, S. V. Postolova, A. Y. Mironov, A. Glatz, M. R. Baklanov, and V. M. Vinokur, Europhysics Lett. 97, 17012 (2012).

[46] J. M. Graybeal and M. R. Beasley, Phys. Rev. B 29, 4167 (1984).

[47] A. V. Pogrebnyakov, J. M. Redwing, J. E. Jones, X. X. Xi, S. Y. Xu, Q. Li, V. Vaithyanathan, and D. G. Schlom, Appl. Phys. Lett. 82, 4319 (2003).

[48] H. M. Jaeger, D. B. Haviland, B. G. Orr, and A. M. Goldman, Phys. Rev. B 40, 182 (1989).

[49] T. Orlando, E. McNiff, S. Foner, and M. Beasley, Phys. Rev. B 19, 4545 (1979).

[50] L. Fàbrega, A. Camón, I. Fernández-Martínez, J. Sesé, M. ParraBorderías, O. Gil, R. González-Arrabal, J. L. Costa-Krämer, and F. Briones, Supercond. Sci. Technol, 24, 075014 (2011).

[51] A. Gubin, K. Il'in, S. Vitusevich, M. Siegel, and N. Klein, Phys. Rev. B 72, 064503 (2005).

[52] T. I. Baturina and S. V. Postolova, in International Workshop of Strongly Disordered Superconductors and the SuperconductorInsulator Transition, Villard de Lans, Vercors, French, 2014, http://sit2014.neel.cnrs.fr/workshop_SIT2014/SIT2014.html.
[53] J. M. Graybeal, Phys. B+C 135, 113 (1985).

[54] Q. Zhao, A. McCaughan, F. Bellei, F. Najafi, D. De Fazio, A. Dane, Y. Ivry, and K. K. Berggren, Appl. Phys. Lett. 103, 142602 (2013).

[55] Q. Zhao, A. N. McCaughan, A. E. Dane, F. Najafi, F. Bellei, D. De Fazio, K. A. Sunter, Y. Ivry, and K. K. Berggren, Opt. Exp. 22, 24574 (2014).

[56] DataThief version 1.6, http://datathief.org, and B. Tummers (2006).

[57] C. Strunk, C. Sürgers, U. Paschen, and H. Löhneysen, Phys. Rev. B 49, 4053 (1994).

[58] W. Siemons, M. Steiner, G. Koster, D. Blank, M. Beasley, and A. Kapitulnik, Phys. Rev. B 77, 174506 (2008).

[59] Y. Liu, D. B. Haviland, B. Nease, and A. M. Goldman, Phys. Rev. B 47, 5931 (1993).

[60] I. L. Landau, D. L. Shapovalov, and I. A. Parshin, Pis'ma Zh. Eksp. Teor. Fiz. 53, 250 (1991) [JETP Lett. 53, 263 (1991)].

[61] R. P. Silverman, Phys. Rev. B 16, 2066 (1977).

[62] R. P. Silverman, Phys. Rev. B 19, 233 (1979).

[63] D. G. Naugle, R. E. Glover III, and W. Moorman, Physica 32, 250 (1971).

[64] C. Delacour, L. Ortega, M. Faucher, T. Crozes, T. Fournier, B. Pannetier, and V. Bouchiat, Phys. Rev. B 83, 144504 (2011).

[65] B. H. Müller, T. Schmidt, and M. Henzler, Surf. Sci. 376, 123 (1997).

[66] S. Maekawa and H. Fukuyama, J. Phys. Soc. Japan 51, 1380 (1982).

[67] V. M. Vinokur, T. I. Baturina, M. V Fistul, A. Y. Mironov, M. R. Baklanov, and C. Strunk, Nature 452, 613 (2008). 